\title{
A multisectoral investigation of a neonatal unit outbreak of Klebsiella pneumoniae bacteraemia at a regional hospital in Gauteng Province, South Africa
}

\author{
V Essel, ${ }^{1}$ MB BCh, MMed (Public Health Medicine), FCPHM, DTM\&H; K Tshabalala, ${ }^{1,2}$ MB BCh, Dip HIV Man (SA); \\ G Ntshoe, ${ }^{1,3}$ BSc (Med) Hons, MPH; E Mphaphuli, ${ }^{1,3}$ MB BCh, MPH, Dip HIV Man (SA); G Feller, ${ }^{1}$ MB BCh; A M Shonhiwa, ${ }^{1}$ MSc, MPH; \\ K McCarthy, ${ }^{1} \mathrm{MB}$ BCh, FC Path, DTM\&H; H Ismail, ${ }^{4} \mathrm{PhD}, \mathrm{MPH}$; W Strasheim, ${ }^{4}$ BSc, BSc Hons, MSc; M Lowe, ${ }^{4} \mathrm{PhD}$; \\ O Perovic, ${ }^{4,5}$ MD, DTM\&H, MMed (Microbiol), FC Path; M Hlonipho, ${ }^{6}$ MA, MBA; N P Govender, ${ }^{4,5}$ MB BCh, MMed (Microbiol), \\ FC Path, MSc (Epidemiol), MSc (Mycol), DTM\&H, Dip HIV Man (SA)
}

\author{
${ }^{1}$ Outbreak Response Unit, Division of Public Health Surveillance and Response, National Institute for Communicable Diseases, National Health \\ Laboratory Service, Johannesburg, South Africa \\ ${ }^{2}$ School of Public Health Medicine, Faculty of Health Sciences, University of the Witwatersrand, Johannesburg, South Africa \\ ${ }^{3}$ School of Health Systems and Public Health, Faculty of Health Sciences, University of Pretoria, South Africa \\ ${ }^{4}$ Centre for Healthcare-Associated Infections, Antimicrobial Resistance and Mycoses, National Institute for Communicable Diseases, National \\ Health Laboratory Service, Johannesburg, South Africa \\ ${ }^{5}$ School of Pathology, Faculty of Health Sciences, University of the Witwatersrand, Johannesburg, South Africa \\ ${ }^{6}$ Gauteng Provincial Health Department Infection Prevention and Control Sub-Directorate, Johannesburg, South Africa
}

Corresponding authors: VEssel (viviene@nicd.ac.za), K Tshabalala (drkhanyisile@gmail.com)

\begin{abstract}
Background. Rates of healthcare-associated infections (HAIs) among babies born in developing countries are higher than among those born in resource-rich countries, as a result of suboptimal infection prevention and control (IPC) practices. Following two reported deaths of neonates with carbapenem-resistant Klebsiella pneumoniae bloodstream infections (BSIs), we conducted an outbreak investigation in a neonatal unit of a regional hospital in Gauteng Province, South Africa.

Objectives. To confirm an outbreak of K. pneumoniae BSIs and assess the IPC programme in the neonatal unit.

Methods. We calculated total and organism-specific BSI incidence risks for culture-confirmed cases in the neonatal unit for baseline and outbreak periods. We conducted a clinical record review for a subset of cases with K. pneumoniae BSI that had been reported to the investigating team by the neonatal unit. An IPC audit was performed in different areas of the neonatal unit. We confirmed species identification and antimicrobial susceptibility, and used polymerase chain reaction for confirmation of carbapenemase genes and pulsedfield gel electrophoresis (PFGE) for typing of submitted clinical isolates.

Results. From January 2017 to August 2018, 5262 blood cultures were submitted, of which 11\% (560/5 262) were positive. Of 560 positive blood cultures, $52 \%(n=292)$ were positive for pathogenic organisms associated with healthcare-associated BSIs. K. pneumoniae comprised the largest proportion of these cases (32\%; 93/292). The total incidence risk of healthcare-associated BSI for the baseline period (January 2017 - March 2018) was 6.8 cases per 100 admissions, and that for the outbreak period (April - September 2018) was 10.1 cases per 100 admissions. The incidence risk of $K$. pneumoniae BSI for the baseline period was 1.6 cases per 100 admissions, compared with 5.0 cases per 100 admissions during the outbreak period. Average bed occupancy for the entire period was $118 \%$ (range $101-133 \%$ ), that for the baseline period was $117 \%$, and that for the outbreak period was $121 \%$. In a subset of 12 neonates with K. pneumoniae bacteraemia, the median (interquartile range (IQR)) gestational age at birth was 27 (26 - 29) weeks, and the median (IQR) birth weight was 1100 (880 $1425)$ g. Twelve bloodstream and 31 colonising K. pneumoniae isolates were OXA-48-positive. All isolates were genetically related by PFGE analysis ( $89 \%$ similarity). Inadequate IPC practices were noted, including suboptimal adherence to aseptic technique and hand hygiene (57\% overall score in the neonatal intensive care unit), with poor monitoring and reporting of antimicrobial use (pharmacy score 55\%). Conclusions. Overcrowding and inadequate IPC and antimicrobial stewardship contributed to a large outbreak of BSIs caused by genetically related carbapenemase-producing K. pneumoniae isolates in the neonatal unit.
\end{abstract}

S Afr Med J 2020;110(8):783-790. https://doi.org/10.7196/SAMJ.2020.v110i8.14471

Rates of healthcare-associated infections (HAIs) among babies born in hospitals in developing countries are 3 - 20 times higher than among those born in resource-rich countries. ${ }^{[1]}$ While poor infection prevention and control (IPC) practices are most proximally associated with transmission of HAIs and high infection rates, contributing factors include ageing hospital infrastructure, overcrowded neonatal units, inappropriate use of antimicrobial agents and inadequate institutional support for IPC and antimicrobial stewardship programmes. ${ }^{[2]}$
Klebsiella pneumoniae is a common pathogen causing invasive HAIs in neonates. ${ }^{[1]}$ Factors that predispose neonates to $K$. pneumoniae infection include prematurity and low birth weight. ${ }^{[3]}$ The emergence of carbapenem-resistant $K$. pneumoniae in neonatal units has rendered recommended first-line empirical regimens for neonatal sepsis ineffective. ${ }^{[4]}$ OXA-48-producing K. pneumoniae strains, which may remain phenotypically susceptible to carbapenems, can be missed in routine laboratories if not confirmed with molecular testing. ${ }^{[5]}$ 
Adequate surveillance and response systems are necessary components in preventing the excess morbidity and mortality associated with HAIs. ${ }^{[4]}$ However, hospital outbreak investigations are complex owing to a multitude of factors influencing transmission of pathogens. ${ }^{[6]}$ Investigations are often compromised because hospitals lack capacity and responsible personnel lack technical skills. ${ }^{[7]}$ Outbreaks can be prevented or terminated early through partnership between technical experts and hospital management. ${ }^{\text {[7.8] }}$ For example, collaborative involvement of staff members in the development and implementation of targeted IPC interventions improved the effectiveness of an intervention in a prolonged outbreak of extended-spectrum beta-lactamase-producing Enterobacteriaceae in a university hospital intensive care unit (ICU) in France. ${ }^{[8]}$

In August 2018, the National Institute for Communicable Diseases (NICD) was alerted through a media report of two deaths associated with $K$. pneumoniae bloodstream infection (BSI) in the neonatal unit of a regional hospital in Gauteng Province, South Africa (SA). Following a preliminary assessment of culture-confirmed cases of BSI from the unit, the NICD initiated an investigation, in collaboration with the Gauteng Provincial Department of Health and the health facility. The objectives of the investigation were to confirm an outbreak of $K$. pneumoniae infection and to assess the IPC programme implementation in the hospital. In addition, prospective microbiological testing and analysis of clinical isolates were performed.

\section{Methods \\ Setting}

The regional hospital where this investigation was conducted provides both inpatient and outpatient services and has a capacity of 821 beds, 61 of which are allocated for neonates. The neonatal unit has 16 beds for babies weighing $<2500 \mathrm{~g}, 4$ beds in both the ICU and the highcare unit, 2 isolation rooms with 1 bed each, 8 beds for kangaroo mother care and 27 beds for stable neonates weighing $>2500 \mathrm{~g}$. The hospital receives referrals from midwife obstetric units in the district, from a local district hospital and from private health facilities.

\section{Investigation}

A four-tiered approach was used for this multisectoral collaborative investigation, with the investigating team comprising representatives from the NICD, the National Health Laboratory Service (NHLS) Infection Control Service Laboratory (ICSL), the Gauteng provincial IPC unit and the hospital. A case was defined as any infant admitted to the neonatal unit with any of the following bacterial or fungal pathogens isolated from blood culture: pathogens from the ESKAPE group (Enterococcus faecium, Staphylococcus aureus, K. pneumoniae, Acinetobacter baumannii, Pseudomonas aeruginosa and Enterobacter spp.), and other organisms considered to be associated with the healthcare environment (Escherichia coli, Stenotrophomonas maltophilia, Serratia marcescens and Candida spp.). We were unable to classify the cases into those with early- or late-onset sepsis because admission dates were not provided.

In order to confirm an outbreak of K. pneumoniae bacteraemia, baseline rates of $K$. pneumoniae BSI and other BSI were calculated using retrospectively collected laboratory data for the period 1 January 2017 through to 31 August 2018. These data were obtained at the time of the investigation from the NICD/NHLS data warehouse that archives NHLS laboratory test information. Corresponding denominator data including admissions, patient-days and number of neonatal deaths (all causes) by month were requested from the hospital. Isolates were not available to confirm carbapenem resistance for the vast majority of these retrospectively identified cases, so we only report overall rates for K. pneumoniae bacteraemia.

A clinical record review was then conducted for a subset of cases with $K$. pneumoniae bacteraemia using a structured case investigation form that included demographic, clinical risk factor and outcome variables. This subset was limited to cases that had been reported to the NICD by the neonatal unit for the period 1 August 2018 through to 13 September 2018.

An IPC audit was performed using the National Department of Health's standardised infection control assessment tool. ${ }^{[9]}$ Nine of the 22 available modules in the tool were chosen according to their relevance to complete a targeted, problem-focused assessment. This included an assessment of IPC practices in the neonatal unit and the hospital's IPC programme. The following modules were used: (i) infection control programme; (ii) pharmacy; (iii) ICUs; (iv) hand hygiene; $(v)$ intravenous fluids and medication; ( $v i$ ) urinary catheters; (vii) intravenous catheters; (viii) facility check list: alcohol hand antiseptic; and (ix) facility check list: hand-washing supplies. The investigating team administered module questionnaires to selected staff members of the hospital. Each module was scored by adding the total number of points for responses. The overall quality of practices measured was translated into three categories: $\mathrm{A}=$ recommended practices are followed consistently and thoroughly ( $>75 \%$ of possible points); $\mathrm{B}=$ recommended practices usually followed (50-75\% of possible points); and $\mathrm{C}=$ training and follow-up needed on recommended practices ( $<50 \%$ of possible points). In addition, an assessment of hand hygiene practices was done by direct observation using a World Health Organization (WHO) tool. ${ }^{[10]}$ Staff in the neonatal unit were observed on one occasion on 5 September 2018. The session lasted 20 - 30 minutes. Hand hygiene opportunities included five indications: (i) before touching a patient; (ii) before clean/aseptic procedure; (iii) after body fluid exposure risk; (iv) after touching a patient; and $(v)$ after touching a patient's surroundings. Adherence was calculated by dividing the total number of hand hygiene actions by the total number of opportunities. A missed opportunity was defined as no hand hygiene action being performed when indicated. Investigators were trained to use the audit tools.

Finally, laboratory investigations were conducted at three sites. Initial diagnostic testing was performed at the local NHLS microbiology laboratory following standard operating procedures for bacterial culture and identification and antimicrobial susceptibility testing. Blood cultures that yielded growth of K. pneumoniae were referred to the NHLS ICSL, where carbapenem minimum inhibitory concentrations (MICs) were determined by Etest (bioMérieux, France), and a modified Hodge test and imipenem v. imipenem + ethylenediaminetetra-acetic acid (EDTA) disc diffusion tests were performed. Suspected carbapenemase-producing K. pneumoniae isolates were referred to the NICD. Rectal swab colonisation surveys were conducted by the hospital IPC staff at different time points from July 2018 through to September 2018, and swabs were sent to the NICD for processing. Swabs were inoculated on MacConkey agar, and the inoculum was streaked out for single colonies and incubated aerobically at $35^{\circ} \mathrm{C}$ for 24 hours. Isolates were identified using a matrixassisted laser desorption/ionisation time-of-flight mass spectrometer (MALDI-TOF MS) instrument (Bruker, USA). Colonies identified as $K$. pneumoniae were subcultured and incubated for 24 hours. The antimicrobial susceptibility profiles were determined with the Microscan Walkaway System (NM44, Beckman Coulter, USA) and results were interpreted using a Clinical and Laboratory Standards Institute (CLSI) guideline. ${ }^{[1]}$ Colistin MIC results were interpreted using a European Committee on Antimicrobial Susceptibility Testing 
(EUCAST) guideline. ${ }^{[12]}$ A subset of $K$. pneumoniae isolates (12 from cases of bacteraemia that had been reported to the NICD by the neonatal unit for the period 1 August 2018 through to 13 September 2018, and 31 from the rectal swab colonisation surveys) underwent molecular testing for the presence of carbapenemase genes $\left(b l a_{\mathrm{VIM}}\right.$, $b l a_{\mathrm{NDM}}, b l a_{\mathrm{OXA}-48}$ and variants, $b l a_{\mathrm{KPC}}, b l a_{\mathrm{GES}}$ and $\left.b l a_{\mathrm{IMP}}\right)$ and molecular strain typing using pulsed-field gel electrophoresis. ${ }^{[13-15]}$ In addition, four historical $K$. pneumoniae isolates that had been submitted by the same hospital to the NICD during the baseline period were also included for comparison.

Data were manipulated and analysed in Excel 2010 (Microsoft, USA) and Stata 13 (StataCorp, USA), respectively.

The University of the Witwatersrand Human Research Ethics Committee approved the urgent outbreak investigation (ref. no. M160667) and the hospital granted written permission to conduct the outbreak investigation (ref. no. 23/08/2016).

\section{Results \\ Baseline rates of BSI}

From 1 January 2017 through to 31 August 2018, 5262 blood cultures were submitted by the neonatal unit. A bacterial or fungal organism was cultured from $11 \%$ of specimens (560/5 262). Of the 560 positive blood cultures, 292 (52\%) yielded pathogens from the ESKAPE group or organisms that are associated with healthcare-associated BSIs (as per the case definition). The other 268 organisms included Klebsiella spp. other than K. pneumoniae, Listeria monocytogenes, non-typhoidal Salmonella spp., Shigella spp. and Streptococcus spp. Among the 292 positive blood culture specimens, $K$. pneumoniae was the most common pathogen $(32 \%$; 93/292), followed by A. baumannii (19\%; 56/292) and S. aureus (13\%; 37/292) (Fig. 1). Forty-seven cases of K. pneumoniae BSI occurred during the baseline period (1 January 2017 - 31 March 2018), with a median (interquartile range (IQR)) of $2(1-5)$ cases per month. A clear increase in the number of cases of $K$. pneumoniae occurred during the outbreak period (1 April 2018 - 13 September 2018; $n=51$ ) compared with the baseline period, despite the number of admissions remaining stable (Fig. 2). From 1 January 2017 to 31 August 2018, there were 3836 neonatal admissions. The overall incidence risk of healthcare-associated BSI for the entire period was 7.6 cases per 100 admissions. The incidence risk of healthcare-associated BSI for the baseline period was 6.8 cases per 100 admissions, and that for the outbreak period was 10.1 cases per 100 admissions (incidence risk ratio 1.5) (Table 1). The overall incidence risk of $K$. pneumoniae BSI was 2.4 cases per 100 admissions; the incidence risk was 1.6 cases per 100 admissions during the baseline period, and 5.0 cases per 100 admissions during the outbreak period (incidence risk ratio 3.1) (Table 2). During August 2018, the number of neonates admitted to the 61-bed unit ranged from 72 to 90 per day, with a total of 217 admissions for that month. The average bed occupancy for the entire period was $118 \%$, ranging from $101 \%$ in March 2018 to a peak of $133 \%$ in August 2018. Average bed occupancy for the baseline period was $117 \%$, and that for the outbreak period was $121 \%$ (Table 1).

\section{Clinical record review}

Of the 51 culture-confirmed cases of K. pneumoniae bacteraemia identified in the neonatal unit from 1 April 2018 to 13 September 2018 , only $24 \%(n=12)$ were reported in a line list to the investigating team by 13 September 2018. These 12 neonates had K. pneumoniae bacteraemia caused by strains that were resistant to carbapenems and had been diagnosed from 1 July 2018 to 13 September 2018. For these 12 neonates, the median (IQR) gestational age at birth was 27 (26 - 29) weeks and the median (IQR) birth weight was 1100 (880 $1425) \mathrm{g}$. Females accounted for 6 of the cases, and 4 neonates were born to mothers who were known to be HIV-seropositive. By the end of the investigation period, 6 neonates had been discharged and the remaining 6 had died. All but one were admitted into the neonatal unit on the day of birth. Reasons for admission included low birth weight, respiratory distress (80\%), hyaline membrane disease (20\%), necrotising enterocolitis (20\%), neonatal sepsis $(20 \%)$ and neonatal jaundice (10\%). The median (IQR) age of the 12 neonates at the time of the first blood culture was $3(1-10)$ days. The majority of neonates had at least one invasive medical device in situ during the course

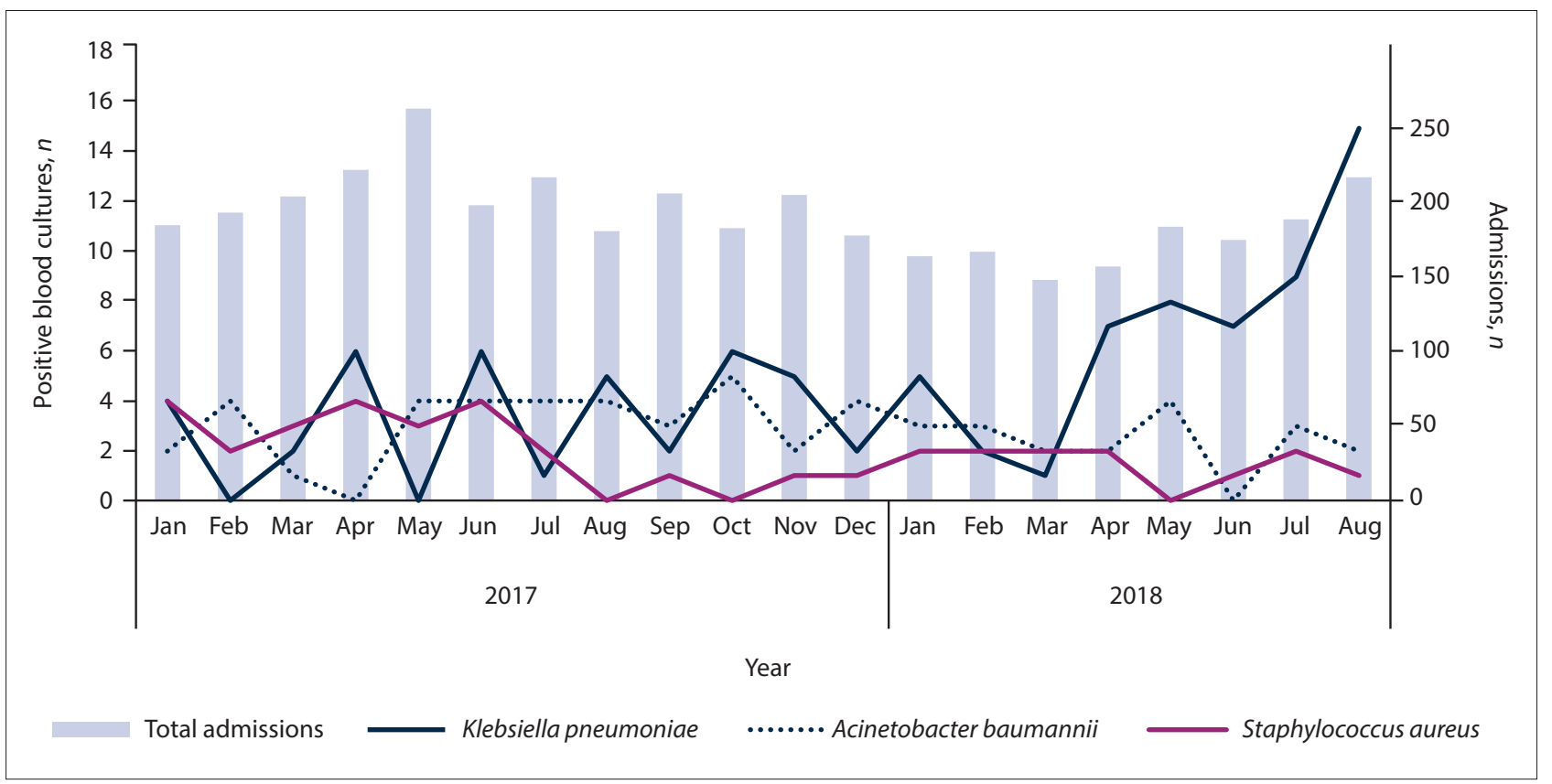

Fig. 1. Number of positive blood cultures of Klebsiella pneumoniae, Acinetobacter baumannii and Staphylococcus aureus, and number of admissions in the neonatal unit of the regional hospital, January 2017 through to August 2018. 


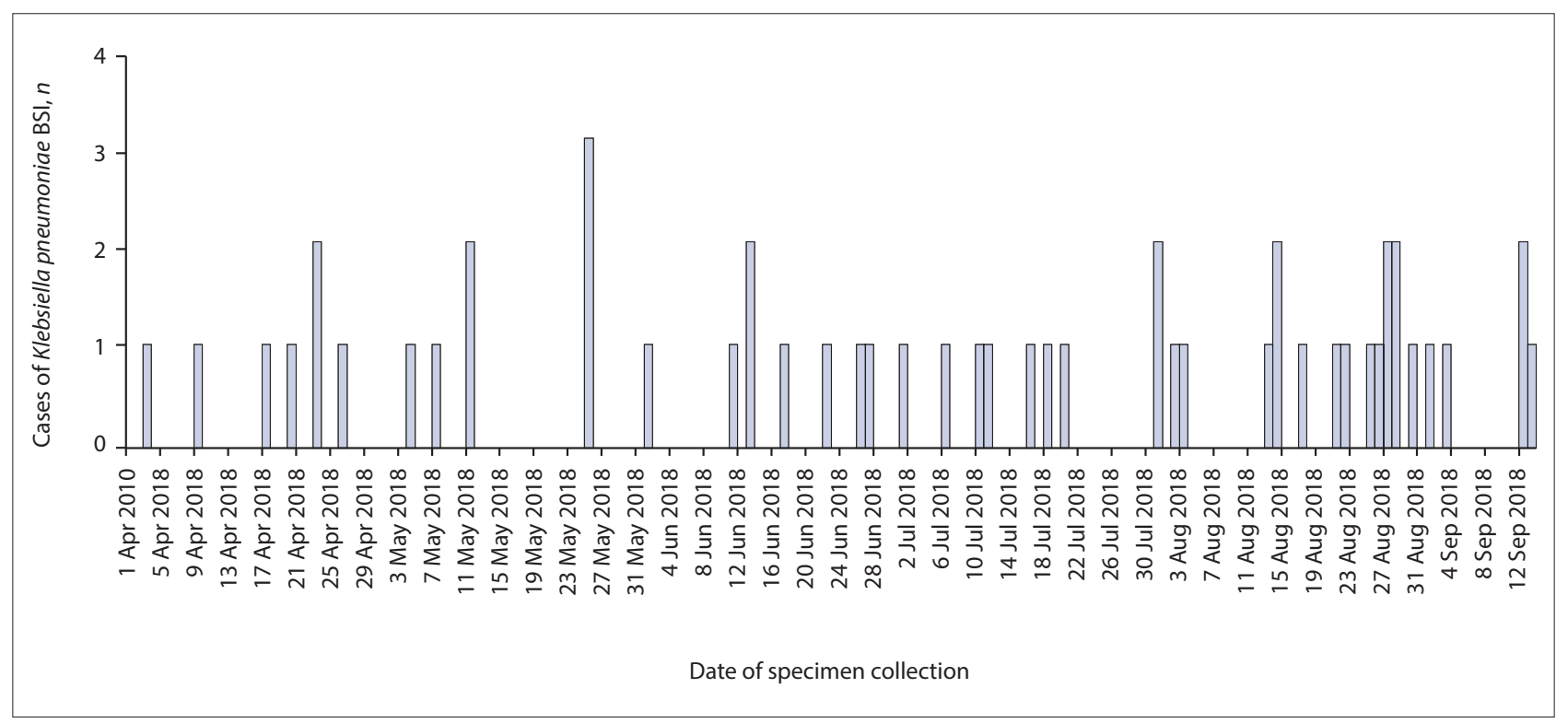

Fig. 2. Epidemic curve of Klebsiella pneumoniae bacteraemia by date of specimen collection, 1 April 2018 through to 13 September 2018 ( $\mathrm{n}=51)$. (BSI = bloodstream infection.)

Table 1. Neonatal unit statistics, proportion of positive blood cultures, and incidence risk of BSI in the neonatal unit of the regional hospital, January 2017 through to August 2018

\begin{tabular}{lllllll}
\hline Year & Month & Admissions, $\boldsymbol{n}$ & Patient-days, $\boldsymbol{n}$ & Bed occupancy, \% & $\begin{array}{l}\text { Positivity for all } \\
\text { blood cultures, \% }\end{array}$ & $\begin{array}{l}\text { Incidence risk of BSI, } \\
\text { cases per 100 admissions }\end{array}$ \\
\hline 2017 & Jan & 185 & 2048 & 108 & 7 & 8.1 \\
& Feb & 193 & 1905 & 112 & 10 & 6.2 \\
& Mar & 204 & 2164 & 114 & 7 & 5.4 \\
& Apr & 222 & 2431 & 133 & 10 & 7.7 \\
& May & 263 & 2480 & 131 & 11 & 5.3 \\
& Jun & 198 & 2285 & 125 & 12 & 7.4 \\
& Jul & 2306 & 122 & 12 & 8.3 \\
& Aug & 181 & 1924 & 102 & 9 & 3.9 \\
& Sep & 217 & 2161 & 118 & 5 & 8.2 \\
& Oct & 183 & 2201 & 116 & 13 & 6.9 \\
& Nov & 205 & 262 & 124 & 8 & 9.1 \\
& Dec & 178 & 2357 & 125 & 11 & 5.4 \\
& Jan & 164 & 2089 & 110 & 15 & 6.1 \\
& Feb & 167 & 2006 & 117 & 14 & 10.8 \\
& Mar & 148 & 1909 & 101 & 14 & 9.2 \\
Total & Apr & 157 & 2021 & 110 & 14 & 7.4 \\
BSI = bloodstream infection. & 184 & 2102 & 111 & 14 & 12.2 \\
& May & 2380 & 130 & 8 & 10.6 \\
& Jun & 175 & 2288 & 121 & 13 & 7.6
\end{tabular}

of their admission, including peripheral intravenous lines, central venous catheters, umbilical catheters and endotracheal tubes.

\section{IPC audit}

The overall score for availability of an IPC programme in the hospital was $98 \%$ (Table 3). The health facility had two trained IPC personnel who requested external assistance to investigate the outbreak. The observed adherence to hand hygiene practices during a single IPC audit on 5 September 2018 was 77\% (49/64). The audit detected gaps in practice related to aseptic technique and an unacceptably low overall score of $57 \%$ in the neonatal ICU (Table 3). The overall pharmacy score was 55\%; areas of weakness in the pharmacy module included the antibiotic control programme (score of 35\%) and monitoring and reporting of antibiotic use (score of 13\%) (Table 3).

\section{Laboratory investigations}

Fifteen bloodstream isolates were cultured from 12 (of 51) cases from the outbreak period. Only 12 of these 15 isolates were available 


\begin{tabular}{|c|c|c|c|c|c|c|}
\hline Year & Month & Admissions, $n$ & Patient-days, $n$ & $\begin{array}{l}\text { Incidence risk of } \\
\text { K. pneumoniae BSI, cases } \\
\text { per } 100 \text { admissions }\end{array}$ & $\begin{array}{l}\text { Incidence rate of } \\
\text { K. pneumoniae BSI, cases } \\
\text { per } 1000 \text { patient-days }\end{array}$ & $\begin{array}{l}\text { Proportion of } \\
\text { K. pneumoniae } \\
\text { among all BSIs, } \% \\
\end{array}$ \\
\hline \multirow[t]{12}{*}{2017} & Jan & 185 & 2048 & 2.2 & 2.0 & 27 \\
\hline & Feb & 193 & 1905 & 0.0 & 0.0 & 0 \\
\hline & Mar & 204 & 2164 & 1.0 & 0.9 & 18 \\
\hline & Apr & 222 & 2431 & 2.7 & 2.5 & 35 \\
\hline & May & 263 & 2480 & 0.0 & 0.0 & 0 \\
\hline & Jun & 198 & 2285 & 3.0 & 2.6 & 30 \\
\hline & Jul & 217 & 2306 & 0.5 & 0.4 & 6 \\
\hline & Aug & 181 & 1924 & 2.8 & 2.6 & 33 \\
\hline & Sep & 206 & 2161 & 1.0 & 0.9 & 25 \\
\hline & Oct & 183 & 2201 & 3.3 & 2.7 & 40 \\
\hline & Nov & 205 & 2262 & 2.4 & 2.2 & 42 \\
\hline & Dec & 178 & 2357 & 1.1 & 0.8 & 18 \\
\hline \multirow[t]{8}{*}{2018} & Jan & 164 & 2089 & 3.0 & 2.4 & 33 \\
\hline & Feb & 167 & 2006 & 1.2 & 1.0 & 22 \\
\hline & Mar & 148 & 1909 & 0.7 & 0.5 & 11 \\
\hline & Apr & 157 & 2021 & 4.5 & 3.5 & 41 \\
\hline & May & 184 & 2102 & 4.3 & 3.8 & 47 \\
\hline & Jun & 175 & 2380 & 4.0 & 2.9 & 54 \\
\hline & Jul & 189 & 2288 & 4.8 & 3.9 & 39 \\
\hline & Aug & 217 & 2514 & 6.9 & 6.0 & 65 \\
\hline Total & & 3836 & 43833 & 2.4 & 2.1 & 32 \\
\hline
\end{tabular}

for molecular testing at the NICD. All 12 bloodstream isolates were positive for $b l a_{\text {OXA-48 }}$ and variants. Two of the 3 isolates not available for molecular testing were modified Hodge testpositive. All 12 isolates were susceptible to amikacin, 11 isolates were susceptible to tigecycline, and 10 isolates were susceptible to colistin, tetracycline and levofloxacin. The 2 colistin-resistant isolates were not confirmed with other phenotypic assays. All isolates were resistant to amoxicillin-clavulanate, ampicillin/ sulbactam, ampicillin, fosfomycin, piperacillin/tazobactam and piperacillin. Four bloodstream isolates, which had been submitted during the baseline period, were positive for $b l a_{\mathrm{OXA}-48}$ and variants; one isolate harboured both the $b l a_{\mathrm{NDM}}$ and $b l a_{\mathrm{OXA}-48}$ and variants genes. Of the 90 rectal swabs submitted by the regional hospital, 31 cultured K. pneumoniae, all of which were positive for $b l a_{\text {OXA-48 }}$ and variants. Three patients with a positive colonisation swab also had K. pneumoniae bacteraemia. Pulsed-field gel electrophoresis analysis indicated that all isolates were genetically related (89\% similarity value), except for a single isolate from a case of bacteraemia diagnosed on 25 August 2018 (strain ML5598: similarity value 79\%) (Fig. 3). Colonising and invasive strains from the outbreak period were genetically related to the four strains from the baseline period (strains ML4343, ML4348, ML4480 and ML4586).

\section{Discussion}

There was a three-fold increase in the incidence risk of $K$. pneumoniae bacteraemia during a 5-month outbreak period in 2018 among patients admitted to the neonatal unit of a large regional hospital, compared with the incidence risk during the preceding 15-month baseline period.

Although retrospectively collected laboratory data confirmed a large number of cases during the outbreak period, only a small subset of cases was reported in a line list at the time of investigation. Further interrogation of these data in collaboration with the hospital staff pointed to gaps in the surveillance and reporting systems in the hospital. As found in a previous study, adequate surveillance and response systems are necessary components in preventing HAIs. ${ }^{[4]}$ Within the reported subset with clinical data, cases were mostly premature neonates with typical risk factors such as low birth weight. ${ }^{[3]}$

K. pneumoniae was the most common pathogen cultured from blood cultures during the outbreak, followed by A. baumannii. We were only able to confirm that 12 of 51 cases during the defined outbreak period were caused by a carbapenem-resistant $K$. pneumoniae strain; isolates were unavailable for the remaining 39 cases. Twelve invasive and 31 colonising K. pneumoniae strains, which were available for testing, carried a carbapenemase-encoding gene ( $b l a_{\text {OXA-48 }}$ and variants) and one isolate had an additional $b l a_{\mathrm{NDM}}$ gene. A systematic review of carbapenemase-producing Enterobacteriaceae (CPE) infections in African countries revealed a rapid increase in the number of reports of CPE infections. ${ }^{[16]}$ This study also demonstrated that OXA-48 and variants was the most commonly reported carbapenemase. ${ }^{[16]}$ Similarly, a study looking at K. pneumoniae isolates in private hospitals across north-eastern SA found OXA-48 and variants to be most common. ${ }^{[17]}$ A high prevalence of carbapenem-resistance genes including $b l a_{\text {OXA-48 }}$ and variants has also been described among multidrug-resistant Gram-negative bacteria in Tanzanian tertiary hospitals. ${ }^{[18]}$ Several outbreaks and sporadic cases of OXA-48 and variants-producing Enterobacteriaceae infections have been reported from European neonatal ICUs, most of which were related to patients with previous exposure to healthcare facilities in Turkey, the Middle East and northern Africa. ${ }^{[19]}$ SA has added CPE to a list of notifiable medical 
Table 3. Results of the IPC audit in the neonatal unit of the regional hospital, 5 September 2018

\begin{tabular}{|c|c|c|}
\hline Module & $\begin{array}{l}\text { Overall score } \\
\text { (category) }^{*}\end{array}$ & Key areas assessed (score) \\
\hline (i) IPC programme & $98 \%(A)$ & $\begin{array}{l}\text { - Infection control regulations accreditation } \\
\text { - Responsibilities and authority } \\
\text { - Infection control committee } \\
\text { - Key infection control personnel } \\
\text { - Infection control education programmes } \\
\text { - Outbreak investigation and nosocomial } \\
\text { infection surveillance }\end{array}$ \\
\hline (ii) Pharmacy & $55 \%(\mathrm{~B})$ & $\begin{array}{l}\text { - Pharmacy key personnel }(100 \%) \\
\text { - Pharmacy services }(84 \%) \\
\text { - Antibiotic control programme }(35 \%) \\
\text { - Antibiotic use monitoring and reporting (13\%) }\end{array}$ \\
\hline (iii) Neonatal ICU & $57 \%(\mathrm{~B})$ & $\begin{array}{l}\text { - ICU staffing (50\%) } \\
\text { - ICU general practices }(50 \%) \\
\text { - Mechanical ventilation }(50 \%) \\
\text { - Prophylaxis and monitoring }(67 \%)\end{array}$ \\
\hline $\begin{array}{l}\text { (iv) Hand hygiene } \\
\text { practices (policy) }\end{array}$ & $70 \%(B)$ & $\begin{array}{l}\text { - Policies on hand hygiene practices in the } \\
\text { clinical area of the neonatal unit }\end{array}$ \\
\hline $\begin{array}{l}\text { ( } v \text { ) Intravenous fluids } \\
\text { and medication }\end{array}$ & $61 \%(B)$ & $\begin{array}{l}\text { - Preparation of intravenous fluids and } \\
\text { medications in the neonatal unit }\end{array}$ \\
\hline (vi) Urinary catheters & $66 \%(B)$ & $\begin{array}{l}\text { - Types of urinary catheters used }(100 \%) \\
\text { - Procedures for use of indwelling urinary } \\
\text { catheters ( } 47 \%) \\
\text { - Procedures for insertion and maintenance of } \\
\text { urinary catheters }(90 \%)\end{array}$ \\
\hline $\begin{array}{l}\text { (vii) Intravenous } \\
\text { catheters }\end{array}$ & $72 \%(B)$ & $\begin{array}{l}\text { - This section assessed the use and care of } \\
\text { intravenous catheters in the neonatal unit }\end{array}$ \\
\hline $\begin{array}{l}\text { (viii) Facility check list: } \\
\text { alcohol hand antiseptic }\end{array}$ & $89 \%(\mathrm{~A})$ & $\begin{array}{l}\text { Different wards or clinical areas were assessed } \\
\text { for the presence of alcohol-based hand } \\
\text { antiseptic using a standardised checklist form }\end{array}$ \\
\hline $\begin{array}{l}\text { (ix) Facility check list: } \\
\text { hand-washing supplies }\end{array}$ & $89 \%(\mathrm{~A})$ & $\begin{array}{l}\text { Different wards or clinical areas were assessed } \\
\text { for the presence of hand-washing supplies } \\
\text { using a standardised checklist form }\end{array}$ \\
\hline $\begin{array}{l}(x) \text { Hand hygiene } \\
\text { (observation) }\end{array}$ & $77 \%(\mathrm{~A})$ & $\begin{array}{l}\text { - Before touching a patient }(83 \%) \\
\text { - Before clean/aseptic procedure }(80 \%) \\
\text { - After body fluid exposure risk }(100 \%) \\
\text { - After touching a patient }(75 \%) \\
\text { - After touching patient's surroundings }(64 \%)\end{array}$ \\
\hline
\end{tabular}

Notes

This section assessed the broader hospital IPC programme. The score indicated/suggested that an infection control programme was available and functional.

Recommended practices were followed in some but not all areas.

Recommended practices were followed in some but not all areas.

There was no policy on covering skin lesions and cuts with waterproof dressing. The hand hygiene policy specified keeping fingernails short and/or not using artificial nails or nail extensions.

There was no designated room for preparing medicines. Samples of multi-use medicine vials were not collected and submitted to a laboratory for testing as part of this audit.

Areas for improvement included ensuring a regular supply of indwelling urine catheters.

Areas for improvement included ensuring the availability of appropriate skin antiseptics for the insertion of intravenous catheters.

Recommended equipment and supplies for hand hygiene practices were generally available but not assessed at each point of care.

Recommended equipment and supplies for hand hygiene practices were available.

A total of 64 opportunities were observed for 4 doctors and 10 nurses. Twenty-four hand-washing actions and 25 hand-rub actions were performed by the 14 healthcare members. A difference was observed in the compliance rates between doctors $(89 \% ; 16 / 18)$ and nurses $(72 \% ; 33 / 46)$.

conditions, and laboratories are required to report cases monthly. The situation in SA is similar to that in India. In a large cohort study conducted among neonates born at three tertiary hospitals in India, the incidence of total neonatal sepsis was $14 \%$ and that of culture-positive sepsis was $6 \% .{ }^{[20]}$ The pathogen mix did not differ between early-onset and late-onset sepsis, and two-thirds of the isolates were Gram-negative bacteria. More than half of K. pneumoniae strains were multidrug resistant. In another cohort study conducted in a level 3 neonatal unit in India, neonatal sepsis accounted for two-thirds of total deaths and bacterial isolates caused $74 \%$ of these infections. ${ }^{[21]} \mathrm{K}$. pneumoniae and A. baumannii were the most common bacterial pathogens and were multidrug resistant in the vast majority of cases. More than $80 \%$ of the isolates were carbapenem resistant.

All available $K$. pneumoniae isolates but one in the outbreak we investigated were closely related by molecular typing. The epidemic 


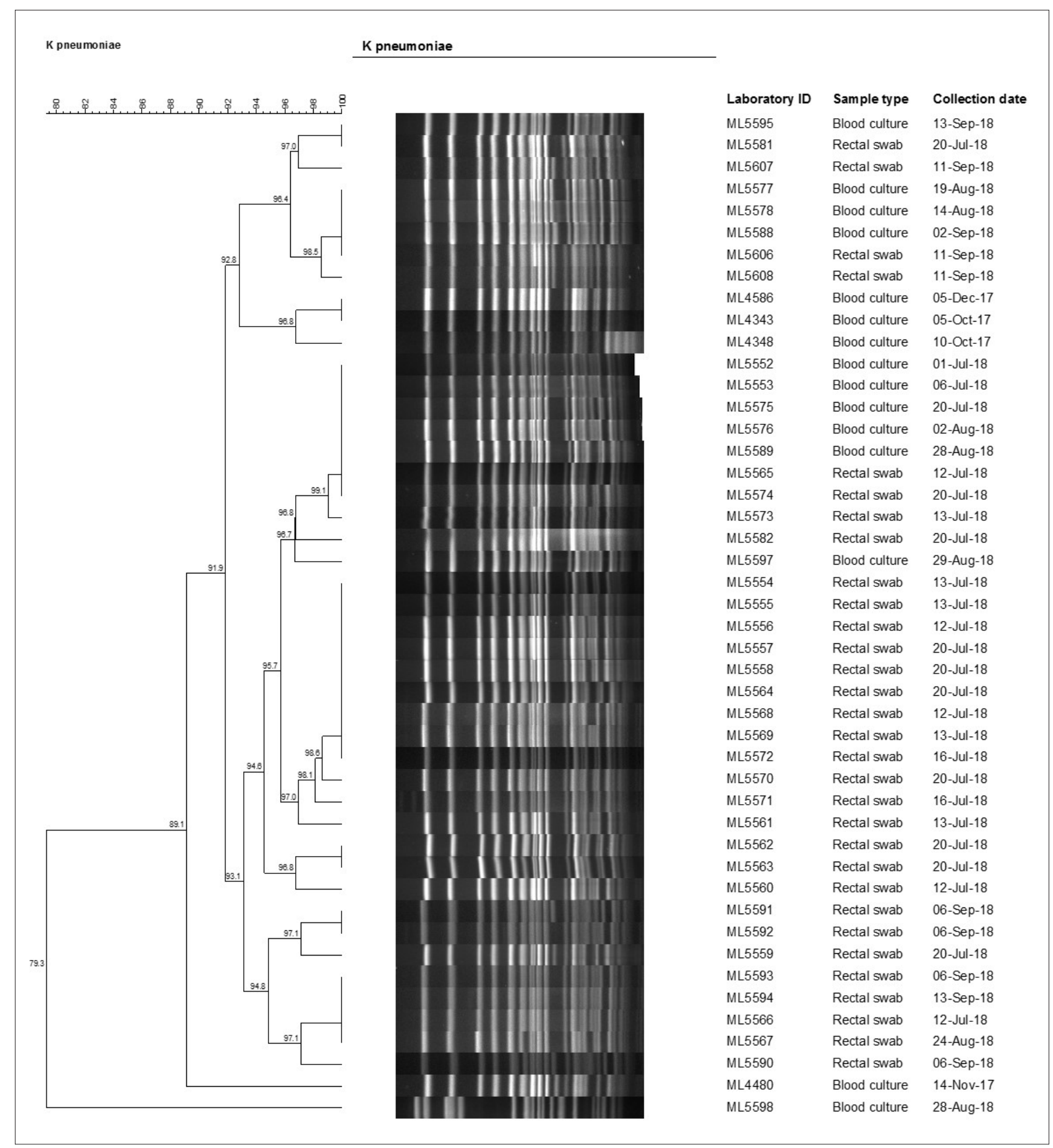

Fig. 3. Pulsed-field gel electrophoresis banding patterns of 15 (invasive) bloodstream and 31 (colonising) rectal swab Klebsiella pneumoniae isolates from the neonatal unit of the regional hospital, 5 October 2017 through to 13 September 2018.

curve and molecular typing results both suggest a propagated outbreak (with person-to-person transmission) of $K$. pneumoniae bacteraemia. The outbreak strains were genetically related to strains from the baseline period, suggesting that the same strain had persisted in the unit for months and caused a large outbreak from April 2018 onwards.

Inadequate support for IPC programmes and inappropriate use of antimicrobials are institutional factors contributing to the increase in HAIs. ${ }^{[2]}$ Although the score for the availability of an IPC programme in the hospital was high, there were gaps in the actual implementation of this programme. The overall pharmacy score was 55\%, with low scores in the antibiotic control programme and in the antibiotic use monitoring and reporting modules. Pharmacy has an important role in antimicrobial stewardship and oversight. ${ }^{[22]}$ Antimicrobial stewardship programmes in hospital settings can improve prescribing and infection outcomes. ${ }^{[22]}$ In terms of IPC, the existence of policies and guidelines alone is not sufficient to ensure adoption and implementation. ${ }^{[23]}$ The WHO recommends ongoing education and training of healthcare workers on the policies and guidelines, coupled with continuous monitoring of adherence. ${ }^{[23]}$ 


\section{Study strengths and limitations}

This investigation was a multisectoral collaborative effort. Collaboration with all relevant stakeholders is a necessity in facilitating outbreak investigations, ensuring appropriate facility response and follow-through on recommendations.

The investigation had a number of limitations. There was no available information on changes in IPC practices between the baseline and outbreak periods. We did not obtain information on the total number of deaths in the unit and the number of deaths associated with K. pneumoniae bacteraemia. Comprehensive patient clinical records were unavailable or incomplete. We characterised only a small subset of invasive isolates. Routine colonisation survey methods were not well documented. We could not calculate colonisation prevalence in the unit because swabs were not collected during a defined period. The IPC audit was conducted on a single day and may not reflect daily practices. The audit also omitted an evaluation of the milk kitchen and auxiliary areas, as well as equipment and environmental cleaning practices. Multi-use medicine vial practices were not documented and no samples of multi-use vials were referred for laboratory testing. We did not specifically document the presence of alcohol-based hand rub at each point of care. We did not observe procedures such as intravenous medicine preparation, line or urinary catheter insertion. No environmental testing was conducted.

\section{Recommendations}

We recommended actions to reduce the high bed occupancy, including closure of the unit to new admissions during the outbreak period. Isolation and/or cohorting of infected or colonised patients and continued implementation of contact precautions were advised. We recommended weekly observations and audits of hand hygiene practices, re-training of all unit personnel and the provision of appropriate equipment and supplies for hand hygiene and aseptic practices. Other recommendations included active surveillance of BSIs in the neonatal unit and the institution of a neonatal antimicrobial stewardship programme. A repeat IPC audit including all auxiliary areas such as the milk kitchen, as well as testing multiuse vials, was suggested. We also recommended a thorough review of environmental and equipment cleaning practices in the unit. We did not recommend relocation of critically ill patients to other hospitals. Although the number of cases decreased in the aftermath of the reported outbreak following implementation of recommended interventions, sporadic outbreaks have continued to occur in this unit.

\section{Conclusions}

Overcrowding with consequential breaches in IPC practices related to hand hygiene and poor aseptic technique contributed to horizontal transmission of carbapenemase-producing and genetically related $K$. pneumoniae strains, resulting in an outbreak of BSIs in the neonatal unit of this regional hospital. Hospitals should conduct active surveillance of neonatal infections and ensure that IPC and antimicrobial stewardship programmes are initiated or strengthened.

\section{Declaration. None.}

Acknowledgements. We thank the Gauteng Provincial Department of Health and the NHLS ICSL for supporting this outbreak investigation, and the staff of the regional hospital for assisting in data collection. We also thank Nevashan Govender of the NICD for alerting NICD staff members to the initial media report.

Author contributions. NPG, VE and KT collected and analysed data, and wrote the manuscript. GN, EM, GF, AMS and HI collected and analysed data. MH collected data and provided support to the hospital staff. WS, $\mathrm{ML}$ and $\mathrm{OP}$ tested the isolates and analysed laboratory data. All authors reviewed the manuscript. KM reviewed the manuscript. NPG reviewed and edited the manuscript.

Funding. None.

Conflicts of interest. None.

1. Zaidi AK, Huskins WC, Thaver D, Bhutta ZA, Abbas Z, Goldmann DA. Hospital-acquired neonatal infections in developing countries. Lancet 2005;365(9465):1175-1188. https://doi.org/10.1016/S01406736(05)71881-X

2. Gill C, Mantaring J, Macleod W, et al. Impact of enhanced infection control at 2 neonatal intensive care units in the Philippines. Clin Infect Dis 2009;48(1):13-21. https://doi.org/10.1086/594120

3. Shane A, Sanchez PJ, Stoll BJ. Neonatal sepsis. Lancet 2017;390(10104):1770-1780. https://doi. org/10.1016/S0140-6736(17)31002-4

4. Dramowski A, Cotton MF, Whitelaw A. A framework for preventing healthcare-associated infection in neonates and children in South Africa. S Afr Med J 2017;107(3):192-195. https://doi.org/10.7196/ SAMJ.2017.v107i3.12035

5. Nazik H, Ongen B, Ilktac M, et al. Carbapenem resistance due to $b l a_{0 X \mathrm{~A} A \mathrm{4}}$ among ESBL-producing Escherichia coli and Klebsiella pneumoniae isolates in a university hospital, Turkey. Southeast Asian J Trop Escherichia coli and Klebsiella pneumoniae isolates in a university hospital, Turkey. Southeast Asian J Trop
Med Public Health 2012;43(5):1178-1185. https://pdfs.semanticscholar.org/65af/5c834dcae5352ff4c79b8 Med Public Health 2012;43(5):1178-1185. https://pdfs.

6. Sood G, Perl T. Outbreaks in healthcare settings. Infect Dis Clin 2016;30(3):661-687. https://doi. 6. Sood G, Perl T. Outbreaks in
org/10.1016/j.idc.2016.04.003

7. Archibald LK, Jarvis WR. Health-care associated infection outbreak investigations by the Centre for Archibald LK, Jarvis WR. Health-care associated infection outbreak investigations by the Centre for
Disease Control and Prevention, 1946 - 2005. Am J Epidemiol 2011;174(11 Suppl):S47-S64. https://doi.

8. Lucet J, Decre D, Fichelle A, et al. Control of a prolonged outbreak of extended-spectrum beta-lactamase producing Enterobacteriaceae in a university hospital. Clin Infect Dis 1999;29(6):1411-1418. https://doi. $\operatorname{org} / 10.1086 / 313511$

9. National Department of Health, South Africa. A Standardised Approach to Improving Hospital Infection Control Practices. Pretoria: NDoH, 2013. http://siapsprogram.org/wp-content/uploads/2014/05/14-072ICAT.final_.pdf (accessed 29 August 2018).

10. World Health Organization. Hand hygiene observation tool. 2009. http://www.who.int/gpsc/5may/tools/ en/ (accessed 4 September 2018).

11. Clinical and Laboratory Standards Institute. Performance Standards for Antimicrobial Susceptibility Testing. 28th ed. CLSI supplement M100. Wayne, Pa.: CLSI, 2018. https://clsi.org/media/1930/ m100ed28_sample.pdf (accessed 4 January 2019).

12. European Committee on Antimicrobial Susceptibility Testing. Breakpoint tables for interpretation of MICs and zone diameters (version 9). 2019. http://www.eucast.org/fileadmin/src/media/PDFs/ EUCAST_files/Breakpoint_tables/v_9.0_Breakpoint_Tables.pdf (accessed 10 January 2019).

13. Singh-Moodley A, Perovic O. Antimicrobial susceptibility testing in predicting the presence of carbapenemase genes in Enterobacteriaceae in South Africa. BMC Infect Dis 16(1):536-546. https://doi, org/10.1186/s12879-016-1858-7

14. Tenover FC, Arbeit RD, Goering RV, et al. Interpreting chromosomal DNA restriction patterns produced by pulsed-field gel electrophoresis: Criteria for bacterial strain typing. I Clin Microbiol 1995;33(9):2233-2239. https://www.ncbi.nlm.nih.gov/pmc/articles/PMC228385/pdf/332233.pdf (accessed 7 January 2019).

15. Ribot EM, Fair MA, Gautom R, et al. Standardization of pulsed-field gel electrophoresis protocols for the subtyping of Escherichia coli O157:H7, Salmonella, and Shigella for PulseNet. Foodborne Pathog Dis 2006;3(1):59-67. https://doi.org/10.1089/fpd.2006.3.59

6. Manenzhe RI, Zar HJ, Nicol MP, Kaba M. The spread of carbapenemase-producing bacteria in Africa: A systematic review. J Antimicrob Chemother 2015;70(1):23-40. https://doi.org/10.1093/jac/dku356 17. Lowe M, Kock MM, Coetzee J, et al. Klebsiella pneumoniae ST307 with bla 2016. Emerg Infect Dis 2019;25(4):739-747. https://doi.org/10.3201/eid2504.181482
20141 18. Mushi MF, Mshana SE, Imirzalioglu C, Bwanga F. Carbapenemase genes among multidrug resistant
Gram negative clinical isolates from a tertiary hospital in Mwanza, Tanzania. Biomed Res Int Gram negative clinical isolates from a tertiary hospit

19. Giuffrè $\mathrm{M}$, Geraci $\mathrm{DM}$, Bonura $\mathrm{C}$, et al. The increasing challenge of multidrug-resistant Gram-negative bacilli: Results of a 5-year active surveillance program in a neonatal intensive care unit. Medicine (Baltimore) 2016;95(10):e3016. https://doi.org/10.1097/md.0000000000003016

20. Investigators of the Delhi Neonatal Infection Study (DeNIS) collaboration. Characterisation and antimicrobial resistance of sepsis pathogens in neonates born in tertiary care centres in Delhi, India: A cohort study. Lancet Glob Health 2016; 4(10):e752-e760. https://doi.org/10.1016/s2214-109x(16)30148-6 21. Jajoo M, Manchanda V, Chaurasia S, et al. Alarming rates of antimicrobial resistance and fungal sepsis in outborn neonates in North India. PLoS ONE 2018;13(6):e0180705. https://doi.org/10.1371/journal. pone. 0180705

22. Wagner B, Filice GA, Drekonja D, et al. Antimicrobial stewardship programs in inpatient hospital settings: A systematic review. Infect Control Hosp Epidemiol 2014;35(10):1209-1228. https://doi. org $/ 10.1086 / 678057$

23. Storr J, Twymann A, Zingg W, et al. Core components for effective infection prevention and control programmes: New WHO evidence-based recommendations. Antimicrob Resist Infect Control 2017;6(6):1-18. https://doi.org/10.1186/s13756-016-0149-9

Accepted 19 February 2020. 